Presented in 2014 IEEE International Conference on Acoustics, Speech and Signal Processing (ICASSP), Florence (Italy), April 2014. DOI: 10.1109/ICASSP.2014.6854507

This work is under IEEE copyright and its IEEE published version may be found in http://www.ieeexplore.ieee.org. This file constitutes the draft version.

Work retroactively supported by project TEC2013-47020-C2-2-R (Ministerio de Economia y Competitividad - SPAIN), published in 2014 before the project's official awarding date

\title{
INTERFERENCE POWER RECYCLING IN TWO-SCALE AD HOC WIRELESS NETWORKS
}

\author{
Javier Villares, Josep Sala, Francesc Rey \\ Signal Theory and Communications Department, Technical University of Catalonia (UPC) \\ Campus Nord UPC, Jordi Girona 1-3, 08034 Barcelona, Spain \\ E-mail: \{javier.villares, josep.sala, francesc.rey\}@upc.edu
}

\begin{abstract}
A two-scale ad hoc wireless network is envisaged in which the interference power generated in long-range connections is recycled by the surrounding wireless nodes in order to support their own short-range communications. We prove that the network energy efficiency is independent of the transmitters range and, therefore, that long-range transmitters can supply the power required for short-range connectivity without degrading the overall network energy efficiency. As an example, we have studied the feasibility of a short-range multipleaccess return-link powered by the interference harvested from neighboring long-range connections.
\end{abstract}

Index Terms - Energy harvesting, wireless power transfer, random wireless networks, multiuser channels

\section{INTRODUCTION AND RELATED WORK}

In the last few years, the energy cost of communications and computing has arisen as a major aspect to be taken into account [1][2]. In this context, energy harvesting has been proposed as a means of scavenging energy from the environment allowing low-power transmitters to operate autonomously without any external power supply [3]. In particular, far-field $\mathrm{RF}$ energy harvesting is a promising technology that allows gathering energy from the surrounding interference field using, potentially, the same aerial and RF front-ends that are used for communication [4][5]. The energy collected in this way is scarce but expected to be sufficient for sustaining low-rate and/or short-range wireless communications [6][7].

The main drawback of far-field energy harvesting is that only a minuscule fraction of the transmitted power (e.g., 1 out of $\left.10^{9}\right)$ is actually transferred to the intended receiver because most of the power is lost in the environment due to the limited directivity of RF antennas. To ameliorate this enormous inefficiency, we suggest using the entire wireless network as a distributed array of antennas that collects as much power as possible from active interfering transmissions. In this paper,

This work has been supported by the Catalan and Spanish Governments under projects 2009SGR1236 (AGAUR), DYNACS (TEC2010- 21245C02/TCM), COMONSENS (CONSOLIDER-INGENIO CSD2008-00010) and THOFU (CENIT CEN-20101019) this procedure is denoted interference harvesting. Our main result is that interference harvesting renders the network energy efficiency independent of the transmitters coverage range (Section 2) and, therefore, it provides an additional motivation for considering long-range connections in the design of energy-efficient ad hoc wireless networks [8][9].

Based on this result, a two-scale ad hoc wireless network is envisaged in which long- and short-range connections coexist and wherein the power required for short-range connectivity is completely obtained from the interference power produced by long-range transmissions. To the best of our knowledge, this approach is new in the literature and, in this paper, is denoted interference recycling. As an example, we have studied the case in which the harvested power is recycled to set up a self-powered short-range return link with the same transmitter from which the power was harvested (Section 3), as also considered in [10]. The main conclusion is that the return-link sum-rate capacity is determined by the radius of long-range connections. In particular, there exists a minimum critical radius that is required for achieving a noticeable return-link sum-rate.

\section{ENERGY EFFICIENCY OF LONG-RANGE CONNECTIONS}

In order to assess the energy efficiency of long-range connections, we consider a wireless network/cluster composed of $N$ nodes/users that is deployed randomly within a circle of radius $r$ centered at the origin of coordinates. For $r$ sufficiently large, the node positions near the center follow approximately a 2-dimensional homogeneous Poisson point process of density $\rho=N /\left(\pi r^{2}\right)$ nodes per square meter. We consider an active transmitter at the origin of coordinates (central node) that is transmitting $P_{T}$ Watts towards a distant destination node (remote node) $d$ meters apart (see Fig. 1). The central node can be connected to an end user which is a member of the wireless network $(d<r)$ or to an external receiver with which the wireless network is communicating $(d>r)$.

For simplicity, we consider that all users are equipped with antennas whose beampattern is a spherical sector of vertical and horizontal angular aperture of $\phi$ radians. In this simplified model, the directivity of the antennas is given by 


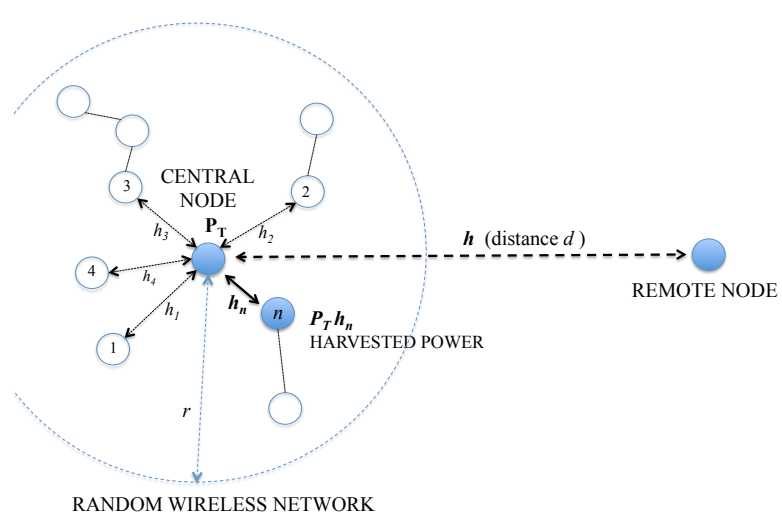

Fig. 1. Studied scenario: a central node of a random wireless network of radius $r$ communicates with a remote node that it is $d$ meters apart, with channel gain $h$. Users near the active transmitter are able to harvest some power from the direct link, which they can use to transmit in the return link (Sec. 3) or to relay the received signal through the wireless network.

$D=2 \pi /(\phi \sin (\phi / 2))$ for $0<\phi \leq \pi$ and their physical size is closely related to their effective area, which is given by $A=D \lambda^{2} / 4 \pi$, with $\lambda$ the signal wavelength.

We adopt the conventional propagation model in which the received power at distance $d>d_{0}$ is computed as $P_{R}=$ $P_{0}\left(d_{0} / d\right)^{\alpha}$, where $\alpha$ is the path-loss exponent, $d_{0}$ the required distance to operate in the far-field and $P_{0}$ the received power at distance $d_{0}$ assuming free-space propagation $(\alpha=2)$, i.e., $P_{0}=P_{T} D^{2}\left(\lambda / 4 \pi d_{0}\right)^{2}=P_{T} d_{0}^{-2} A^{2} / \lambda^{2}$ with $D$ the directivity and $A$ the effective area of both the transmit and receive antennas. This model is adopted for simplicity but the analysis could be extended to more accurate models [11].

According to the model above, the wireless network/cluster is able to harvest the following amount of power:

$$
P_{h}=P_{T} \sum_{n=1}^{N} h_{n}=P_{T} d_{0}^{\alpha-2} \frac{A^{2}}{\lambda^{2}} \sum_{n=1}^{N} a_{n} d_{n}^{-\alpha}
$$

where $h_{n}$ is the gain of the link connecting the transmitter (central node) and the $n$-th receiving node (Fig. 1), $d_{n}$ the corresponding random distance and $a_{n}$ a random variable that indicates whether electromagnetic visibility is sustained between the central node and the $n$-th node. More specifically, $a_{n}=1$ if the transmitter and $n$-th node beams are aligned along the horizontal direction with a pointing error within $\pm \phi / 2$. Otherwise, $a_{n}$ equals zero. We assume for simplicity that all users are in the same horizontal plane and that the vertical pointing error is always within $\pm \phi / 2$. Also, we assume that the antenna azimuth (i.e. horizontal pointing direction) for the $N$ wireless nodes is uniformly distributed between 0 and $2 \pi$. This last assumption is in line with an ad hoc wireless network of randomly associated nodes but it should be reconsidered in other scenarios in which all terminals are presumably pointed in the direction of the central node (e.g., in a mobile cellular network).

Taking all this into account, the harvested power $P_{h}$ is a random variable whose average value is given by

$$
\bar{P}_{h}=\mathbb{E}\left\{P_{h}\right\}=P_{T} d_{0}^{\alpha-2} \frac{A^{2}}{\lambda^{2}} N \mathbb{E}\left\{a_{n}\right\} \mathbb{E}\left\{d_{n}^{-\alpha}\right\}
$$

where $\mathbb{E}\left\{a_{n}\right\}=p\left(a_{n}=1\right)=\phi^{2} /(2 \pi)^{2}$ is the probability of (electromagnetic) visibility between the central node and the $n$-th receiving node and, it is found that: $N \mathbb{E}\left\{d_{n}^{-\alpha}\right\}=$ $\rho \pi r^{2} \int_{d_{0}}^{r} x^{-\alpha} \frac{2 \pi x}{\pi r^{2}} d x=2 \pi \rho d_{0}^{2-\alpha} g(\alpha, r)$ with

$$
g(\alpha, r)=\frac{1-\left(r / d_{0}\right)^{2-\alpha}}{\alpha-2}
$$

for $\alpha>2$. If the wireless network is large enough $\left(r \gg d_{0}\right)$, $g(\alpha, r)$ converges to the constant $1 /(\alpha-2)$ [12], meaning that the power captured by distant users becomes irrelevant. ${ }^{1}$

Finally, if we plug $\mathbb{E}\left\{a_{n}\right\}$ and $N \mathbb{E}\left\{d_{n}^{-\alpha}\right\}$ into (2), we obtain that the average harvested power $P_{h}$ when $r \gg d_{0}$ reads as follows

$$
\bar{P}_{h}=P_{T} K \frac{A \rho}{\alpha-2}
$$

where $\rho=N /\left(\pi r^{2}\right)$ stands for the network density and we have introduced the constant

$$
K=\frac{A \phi^{2}}{2 \pi \lambda^{2}}=\frac{\phi}{4 \pi \sin (\phi / 2)}
$$

which takes values between $K_{\min }=1 /(2 \pi)$ (for $\phi \rightarrow 0$ ) and $K_{\max }=1 / 4$ (for $\phi=\pi$ ). Thus, in the studied set-up, once the effective area $A$ is fixed, the harvested power becomes practically independent of the carrier frequency with variations limited to $10 \log _{10}\left(K_{\max } / K_{\min }\right) \approx 2 \mathrm{~dB}$.

To conclude the analysis, if we focus on $\alpha>2$ and $r \gg$ $d_{0}$, we obtain that the (average) energy efficiency of the wireless network is given by

$$
E(\alpha)=\eta \bar{P}_{h} / P_{T}=\eta K \frac{A \rho}{\alpha-2}
$$

where a generic efficiency factor $\eta$ is introduced to account for the incurred RF/DC power conversion losses [4]. Notice that the power received by the remote node is omitted from (6) because it is negligible compared with $\bar{P}_{h}$ when $d \gg \sqrt{\rho}$.

The obtained network efficiency $E(\alpha)$ agrees correctly with Monte Carlo simulations (Fig. 2) for $r$ sufficiently large and moderate values of $\rho$. However, equation (6) is

\footnotetext{
${ }^{1}$ Notice that if $\alpha=2$ (i.e., free-space propagation), $g(\alpha, r)=\ln \left(r / d_{0}\right)$ is monotonically increasing in $r$, meaning that the contribution of distant users cannot be neglected. In all cases, we only account for the power harvested by far-field users (i.e., $d_{n}>d_{0}$ ) because the adopted propagation model does not apply to near-field communications.
} 


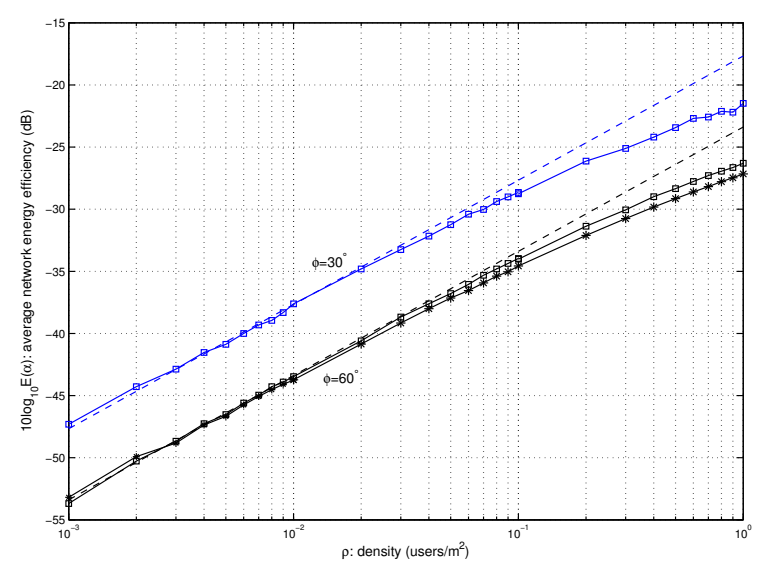

Fig. 2. Average network energy efficiency $(\mathrm{dB})$ as a function of the network density for two different sectorial antennas with $\phi=30^{\circ}, 60^{\circ}$. Dashed curves correspond to the closedform expression derived in (6). The average energy efficiency corresponding to the nearest harvesting node is also plotted with asterisks $(*)$ for $\phi=60^{\circ}$. The other simulation parameters are $d_{0}=2 \mathrm{~m}, d=40 \mathrm{~m}, r=50 \mathrm{~m}, \alpha=3, \eta=0.25$ and carrier frequency $868 \mathrm{MHz}$ (lower ISM band).

not accurate for large values of $\rho$ because the higher the network density $\rho$ the higher the probability of a node being obstructed by other nodes. ${ }^{2}$ As a first approximation, obstructions were simulated in Fig. 2 considering that there is a "cone" of shadow of $\sqrt{A} / d_{n}$ radians behind the $n$-th node, with $n=1, \ldots, N$. In that case, the network energy efficiency $E(\alpha)$ does not increase indefinitely with $\rho$, as wrongly predicted in (6).

So far we have considered that the $N$ nodes contribute to the overall harvested power $\bar{P}_{h}$. However, it happens that most of the power is harvested by a few nodes near the active transmitter. This is especially true when the network density is low. Simulations are presented in Fig. 2 (asteriks, *') confirming that most power is captured by the nearest harvester.

\section{INTERFERENCE RECYCLING FOR SHORT-RANGE CONNECTIVITY: AN EXAMPLE}

In this section, we study how the interference power obtained in Section 2 can be recycled (reused) to set up a return link with the central node (Fig. 1). In theory, all the harvested power in (1) could be recycled if the received signal were processed at the wireless terminals using reversible computing [13][14]. In the realm of reversible computing, the received signal can be decoded without dissipating any energy and, afterwards, the full received power stored in a battery

\footnotetext{
${ }^{2}$ We are assuming however that that the remote node is never obstructed.
}

for upcoming transmissions. On the contrary, in [15][16][17] the problem of simultaneous information and power transfer is addressed taking into account existing constraints of current communication electronics. Although reversible computing is not available in current communication systems, it is adopted hereafter with the aim of determining the theoretical limits of interference-recycling powered communications.

It is worth noting that there exists a non-trivial trade-off between the achievable energy and information transmission rate [13][18][19] since the transmission scheme that maximizes channel capacity is usually not the same that maximizes power transfer. In this section, we will consider that the central node selects the transmission scheme so as to maximize the information rate without imposing any requirement on the transferred energy, as done in [20][21].

In the studied scenario (Fig. 1), the central node transmitted power $P_{T}$ is selected to guarantee a throughput of $R$ bits per channel use (bps/Hz) with the remote node and, at the same time, a non-zero throughput with the neighbouring wireless nodes. To do so, the central node has to assign a small fraction of the transmitted power $\beta P_{T}$ to communicate with the wireless network and the rest of the power $(1-\beta) P_{T}$ to communicate with the remote node. The factor $\beta$ guarantees that the remote node throughput is not reduced in excess when the medium is shared with the wireless network. In particular, we will impose that the remote node throughput is a (large) fraction $\gamma$ of the point-to-point achievable throughput $(\beta=0)$. Accordingly, the transmitted power $P_{T}$ and the constant $\beta$ can be obtained by solving the following two equations:

$$
\begin{aligned}
R & =\log _{2}\left(1+\frac{(1-\beta) \Gamma}{1+\beta \Gamma}\right)=\log _{2}\left(\frac{1+\Gamma}{1+\beta \Gamma}\right) \\
R / \gamma & =\log _{2}(1+\Gamma)
\end{aligned}
$$

where $\Gamma=P_{T} h / P_{N}$ denotes the SNR at the remote node, $P_{N}$ is the noise power of all the receivers and $h$ the channel gain between the central and the remote node ( $d$ meters apart):

$$
h=P_{R} / P_{T}=\left(\frac{A}{\lambda d_{0}}\right)^{2}\left(d / d_{0}\right)^{-\alpha}
$$

Assuming that $h<h_{n}$ (for all $n$ ), equation (7) corresponds to the capacity of the weakest user in a (scalar) Gaussian broadcast channel whereas equation (8) is just the capacity of the point-to-point AWGN channel.

Regarding the wireless network, the achievable throughput depends on how the power $P_{T} \beta$ is distributed over the $N$ wireless nodes as well as on the nodes random position $\left\{d_{n}\right\}_{n=1}^{N}$. In particular, if other constraints are not imposed [20], the maximum sum-rate $R_{S}$ is achieved when the central node assigns the available power $P_{T} \beta$ to the strongest terminal with visibility. Formally, if $h_{\max }=\max \left\{a_{n} h_{n}\right\}$, we obtain that $R_{S} \leq \log _{2}\left(1+\beta \Gamma h_{\max } / h\right)$.

Let us study now the return link in which the remote node and the other $N$ wireless nodes want to communicate simultaneously with the central node. The remote node is assumed 
to be equipped with an external power supply providing a transmitted power of $P_{T}$ Watts, which equals the central node transmitted power. By contrast, the $N$ wireless nodes are supposed to transmit using only the power harvested from the forward link, which is $P_{T} h_{n}$ in the case of the $n$-th node (Fig. 1). The capacity region of the resulting Gaussian multiple access channel is therefore defined by the following three equations:

$$
\begin{aligned}
R_{S}^{\prime} & \leq \log _{2}\left(1+\frac{\Gamma \eta}{h} \sum_{n=1}^{N} a_{n} h_{n}^{2}\right) \\
R^{\prime} & \leq \log _{2}(1+\Gamma) \\
R_{S}^{\prime}+R^{\prime} & \leq \log _{2}\left(1+\Gamma+\frac{\Gamma \eta}{h} \sum_{n=1}^{N} a_{n} h_{n}^{2}\right)
\end{aligned}
$$

where $R^{\prime}$ is the rate from the remote node, $R_{S}^{\prime}$ the wireless network sum rate and $\eta$ the RF/DC power conversion efficiency (6). If we consider a symmetric bi-directional connection between the central and remote nodes (i.e., $R^{\prime}=R$ ) and the penalization factor $\gamma$ is not too low, it follows that we can operate near the boundary of the third equation where the sum-rate of the wireless network is upper bounded as follows:

$$
R_{S}^{\prime} \leq \log _{2}(1+\Gamma g)-R
$$

where $\Gamma=2^{R / \gamma}-1(8)$ and the random gain is defined as $g=$ $1+\frac{\eta}{h} \sum_{n=1}^{N} a_{n} h_{n}^{2}$. Notice that (13) is the maximum returnlink sum-rate that can be attained using the power captured from the forward link for a given realization of $g$.

In a dynamic scenario in which the wireless node positions $d_{n}$ and the antenna azimuths $a_{n}$ evolve in time randomly, the maximum achievable sum-rate $R_{S}^{\prime}$ is determined by the ergodic sum-rate capacity [22], which means that

$$
R_{S}^{\prime} \leq R_{S}^{e r g}=\mathbb{E}_{g}\left\{\log _{2}(1+\Gamma g)\right\}-R .
$$

This equation has been simulated in Fig. 3 as a function of $d$ showing that it grows as $\alpha \log _{2}(d)$ for large values of $d$ whereas it converges to $\frac{1-\gamma}{\gamma} R$ for $d$ going to zero. Accordingly, (14) admits the following piecewise approximation:

$$
R_{S}^{e r g} \lesssim \begin{cases}\frac{1-\gamma}{\gamma} R & d \leq d_{c} \\ \frac{1-\gamma}{\gamma} R+\alpha \log _{2}\left(d / d_{c}\right) & d>d_{c}\end{cases}
$$

where the critical distance $d_{c}$ is obtained by means of Monte Carlo simulations taking into account that

$$
\log _{2}(1+\Gamma g) \approx \alpha \log _{2}(d)+\log _{2}\left(\frac{\Gamma \lambda^{2} \eta}{A^{2} d_{0}^{\alpha-2}} \sum_{n=1}^{N} a_{n} h_{n}^{2}\right)
$$

for large values of $d$.

The ergodic sum-rate capacity (14) is only meaningful when the random gain $g$ is time-varying. Otherwise, in a static or quasi-static scenario, it is preferable to work with the outage sum-rate capacity [22]. Despite outage simulations are not included in the paper due to space limitations, the same asymptotic dependence (15) on the remote node distance $d$ is manifested when dealing with the outage sum-rate.

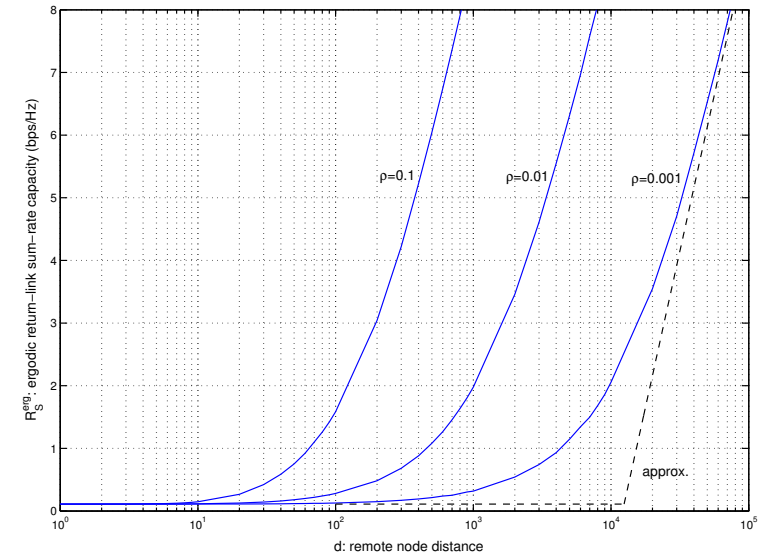

Fig. 3. Return-link ergodic sum-rate capacity as a function of the remote node distance $d$ when the wireless network terminals power is harvested from the forward link (14). An idealized scenario is considered in which the RF/DC power conversion efficiency is $\eta=1$ and reversible computing is implemented at the wireless terminals. Different densities $(\rho)$ have been simulated: $0.001,0.01$ and 0.1 . The throughput of the primary link with the remote node is fixed to $R=1$ $\mathrm{bps} / \mathrm{Hz}$, which is a fraction $\gamma=0.9$ of the achievable rate in the point-to-point case. The rest of parameters have been set to the following values: $d_{0}=2 \mathrm{~m}, r=200 \mathrm{~m}, \alpha=3, \phi=60^{\circ}$ and carrier frequency $868 \mathrm{MHz}$. The dashed curve correspond to the asymptotic approximation in (15) for $\rho=0.001$.

\section{CONCLUSIONS}

We have shown that the energy efficiency of a wireless network is largely enhanced when the wireless terminals are endowed with the capacity of harvesting the interference from surrounding transmissions. In that case, the energy efficiency does not depend on the transmission range but on the wireless network density. This result imposes a revision of existing energy-efficient forwarding/routing strategies and reinforces the interest of long hops in the design of energy-efficient ad hoc wireless networks [8][9]. As shown in this paper, besides reducing the network latency and other benefits, long hops facilitate that the nodes near the transmitter harvest enough power to support their own short-range communications.

As an example, we have studied the case in which the wireless network uses the harvested power to set up a return link with the node whose power was harvested, concluding that there exists a critical hop length that allows achieving a significant return-link sum-rate. Results are presented in terms of the ergodic sum-rate capacity but the same conclusions are valid when dealing with the outage sum-rate capacity in static or quasi-static scenarios. 


\section{REFERENCES}

[1] Congzheng Han, T. Harrold, S. Armour, I. Krikidis, S. Videv, Peter M. Grant, H. Haas, J.S. Thompson, I. Ku, Cheng-Xiang Wang, Tuan Anh Le, M.R. Nakhai, Jiayi Zhang, and L. Hanzo, "Green radio: radio techniques to enable energy-efficient wireless networks," Соттиnications Magazine, IEEE, vol. 49, no. 6, pp. 46-54, 2011.

[2] A. Ephremides, "Energy concerns in wireless networks," Wireless Communications, IEEE, vol. 9, no. 4, pp. 48-59, 2002.

[3] J.A. Paradiso and T. Starner, "Energy scavenging for mobile and wireless electronics," Pervasive Computing, IEEE, vol. 4, no. 1, pp. 18-27, 2005.

[4] T. Le, K. Mayaram, and T. Fiez, "Efficient far-field radio frequency energy harvesting for passively powered sensor networks," Solid-State Circuits, IEEE Journal of, vol. 43, no. 5, pp. 1287-1302, 2008.

[5] Wei Zhao, Kwangsik Choi, S. Bauman, Z. Dilli, T. Salter, and M. Peckerar, "A radio-frequency energy harvesting scheme for use in low-power ad hoc distributed networks," Circuits and Systems II: Express Briefs, IEEE Transactions on, vol. 59, no. 9, pp. 573577, 2012.

[6] H.J. Visser and R.J.M. Vullers, "Wireless sensors remotely powered by RF energy," in Antennas and Propagation (EUCAP), 2012 6th European Conference on, 2012, pp. 1-4.

[7] J. P. Olds and W.K.-G. Seah, "Design of an active radio frequency powered multi-hop wireless sensor network," in Industrial Electronics and Applications (ICIEA), 2012 7th IEEE Conference on, 2012, pp. 17211726.

[8] M. Haenggi and D. Puccinelli, "Routing in ad hoc networks: a case for long hops," Communications Magazine, IEEE, vol. 43, no. 10, pp. 93-101, 2005.

[9] J. Deng, Y. S. Han, P.-N. Chen, and P. K. Varshney, "Optimal transmission range for wireless ad hoc networks based on energy efficiency," Communications, IEEE Transactions on, vol. 55, no. 7, pp. 1439-1439, 2007.

[10] J. Hyungsik and R. Zhang, "Throughput maximization in wireless powered communication networks," Wireless Communications, IEEE Transactions on, vol. 13, no. 1, pp. 418-428, Jan. 2014.

[11] A. Martinez-Sala, J.M. Molina-Garcia-Pardo, E. EgeaLopez, J. Vales-Alonso, L. Juan-Llacer, and J. GarciaHaro, "An accurate radio channel model for wireless sensor networks simulation," Communications and Networks, Journal of, vol. 7, no. 4, pp. 401-407, 2005.

[12] H.S. Lichte, S. Valentin, and H. Karl, "Expected interference in wireless networks with geometric path loss: a closed-form approximation," Communications Letters, IEEE, vol. 14, no. 2, pp. 130-132, 2010.

[13] L.R. Varshney, "On energy/information cross-layer architectures," in Information Theory Proceedings (ISIT), 2012 IEEE International Symposium on, 2012, pp. $1356-1360$.

[14] R. Landauer, Computation, measurement, communication and energy dissipation, Prentice Hall, Ed. Englewood Cliffs, NJ, 1989.

[15] R. Zhang and C.K. Ho, "MIMO broadcasting for simultaneous wireless information and power transfer," Wireless Communications, IEEE Transactions on, vol. 12, no. 5, pp. 1989-2001, 2013.

[16] X. Zhou, R. Zhang, and C.K. Ho, "Wireless information and power transfer: Architecture design and rateenergy tradeoff," in Global Communications Conference (GLOBECOM), 2012 IEEE, 2012, pp. 3982-3987.

[17] Chao Shen, Wei-Chiang Li, and Tsung-Hui Chang, "Simultaneous information and energy transfer: A two-user MISO interference channel case," in Global Communications Conference (GLOBECOM), 2012 IEEE, 2012, pp. 3862-3867.

[18] L.R. Varshney, "Transporting information and energy simultaneously," in Information Theory, 2008. ISIT 2008. IEEE International Symposium on, 2008, pp. $1612-1616$.

[19] P. Grover and A. Sahai, "Shannon meets Tesla: Wireless information and power transfer," in Information Theory Proceedings (ISIT), 2010 IEEE International Symposium on, 2010, pp. 2363-2367.

[20] J. Rubio and A. Pascual-Iserte, "Energy-efficient resource allocation techniques for battery management with energy harvesting nodes: a theoretical approach," in IEEE Wireless Communications and Networking Conference (WCNC), 2013, pp. 795-800.

[21] J. Rubio, A. Pascual-Iserte, and M. Payaro, "Energyefficient resource allocation techniques for battery management with energy harvesting nodes: a practical approach," in Wireless Conference (EW), Proceedings of the 2013 19th European, 2013, pp. 1-6.

[22] D. Tse and P. Viswanath, Fundamentals of wireless communication, Cambridge University Press, New York, NY, USA, 2005. 